University of Nebraska - Lincoln

DigitalCommons@University of Nebraska - Lincoln

Faculty Publications from the Department of Engineering Mechanics

Mechanical \& Materials Engineering,

Department of

2009

\title{
Connected Vibrating Piezoelectric Bimorph Beams as a Wide- band Piezoelectric Power Harvester
}

Zengtao Yang

Central South University, Changsha, Hunan, China

Jiashi Yang

University of Nebraska-Lincoln, jyang1@unl.edu

Follow this and additional works at: https://digitalcommons.unl.edu/engineeringmechanicsfacpub

Part of the Mechanical Engineering Commons

Yang, Zengtao and Yang, Jiashi, "Connected Vibrating Piezoelectric Bimorph Beams as a Wide-band Piezoelectric Power Harvester" (2009). Faculty Publications from the Department of Engineering Mechanics. 77.

https://digitalcommons.unl.edu/engineeringmechanicsfacpub/77

This Article is brought to you for free and open access by the Mechanical \& Materials Engineering, Department of at DigitalCommons@University of Nebraska - Lincoln. It has been accepted for inclusion in Faculty Publications from the Department of Engineering Mechanics by an authorized administrator of DigitalCommons@University of Nebraska - Lincoln. 


\title{
Connected Vibrating Piezoelectric Bimorph Beams as a Wide-band Piezoelectric Power Harvester
}

\author{
Zengtao Yang \\ Institute of Mechanics and Sensing Technology, School of Civil Engineering and Architecture, \\ Central South University, Changsha, Hunan 410083, China \\ Jiashi Yang \\ Department of Engineering Mechanics, University of Nebraska-Lincoln, Lincoln, NE 68588-0526, USA \\ Corresponding author - J. Yang, e-mail jyang1@unl.edu
}

\begin{abstract}
We analyze coupled flexural vibration of two elastically and electrically connected piezoelectric beams near resonance for converting mechanical vibration energy to electrical energy. Each beam is a so-called piezoelectric bimorph with two layers of piezoelectrics. The 1D equations for bending of piezoelectric beams are used for a theoretical analysis. An exact analytical solution to the beam equations is obtained. Numerical results based on the solution show that the two resonances of individual beams can be tuned as close as desired by design when they are connected to yield a wide-band electrical output. Therefore, the structure can be used as a wide-band piezoelectric power harvester.
\end{abstract}

Keywords: piezoelectricity, generator, power harvester

\section{Introduction}

Piezoelectric materials have been used for a long time to make various electromechanical transducers. In particular, due to their strong piezoelectric coupling, polarized ferroelectric ceramics have been used for force or power handling devices including actuators and transformers, etc. Recently, due to the rapid development of wireless electronic devices in both civilian and military applications, operating some of these devices without a wired power source has become an important issue. One approach is to harvest power from the operating environment. Piezoelectric materials are natural candidates for devices that scavenge ambient vibration energy by converting mechanical energy into electric energy, which will then be used for powering small electronic devices of a very low power requirement, (e.g. Cho et al., 2000; Engel et al., 2000; Ha, 2001; Taylor et al., 2001; Roundy et al., 2003; Jiang et al., 2005; Yang et al., 2005). More references can be found in a recent review article by Anton and Sodano, 2007. Such a piezoelectric device used to be called a piezoelectric generator, but a more recent terminology is a piezoelectric power harvester.

Ambient mechanical vibration energy may have various frequency spectra. A piezoelectric power harvester is a resonant device (Yang et al., 2005) in the sense that its electrical output is frequency dependent, and is relatively large near a particular resonant frequency of the harvester structure. Therefore, a specific power har- vester can effectively pick up vibration energy only at a particular frequency. Common beam bimorph flexural mode power harvesters (Cho et al., 2000; Ha, 2001; Jiang et al., 2005) are for relatively low-frequency energy source. Plate or shell thickness mode power harvesters (Yang et al., 2005; Jiang et al., 2006; Yang et al., 2007) are for relatively high frequency applications. There are also spiral-shaped harvesters (Hu et al., 2006; Hu et al., 2007a) and corrugated plate harvesters (Hu et al., 2008) for very low-frequency situations. Various other techniques have also been used for adjusting the vibration characteristics of harvester structures. This includes attaching concentrated masses for their inertial effect on frequency (Jiang et al., 2005; Jiang and Hu, 2007), varying the geometry of the structural member (Hu et al., $2007 \mathrm{~b}$ ), and using mechanical pre-load for frequency adjustment (Hu et al., 2007c).

The above efforts on frequency design are all still based on the resonant frequency of one particular mode. Although power harvesters of different frequencies can be obtained, the resonances are all narrow-band in the sense that they can only collect energy effectively near a particular resonant frequency. In this article, we propose to use connected or coupled bimorph beams whose resonant frequencies are very close to each other and are adjustable. It will be shown through a theoretical analysis that with proper design such a structure is wideband in the sense that it can pick up vibration energy over a wider frequency range than a single-beam harvester structure. 


\section{Structure}

Consider the structure shown in Figure 1. The left ends of the two beams are cantilevered into a wall that is in a vertical, time-harmonic motion with a known amplitude $A$ at a given frequency $\omega$. The right ends of the beams are each connected to a concentrated mass and are connected by a spring with a constant $K$. The concentrated masses are made from heavy materials. Their mass needs to be considered, but they are geometrically small so that their rotatory inertia can be neglected. The two beams are identical. Each beam is what is called a piezoelectric bimorph with a pair of identical ceramic layers as shown in Figure 2. The ceramic layers are shown by the shaded areas, poled along the thickness direction and separated by an elastic layer in the middle. The thick lines in the figure represent electrodes, placed upon the upper and lower surfaces of the piezoelectric layers. The two electrodes in contact with the elastic layer are connected where the electric potential $\varphi$ is taken to be zero as a reference. The very top and very bottom electrodes are also connected. When the beam bends, one piezoelectric layer gets stretched while the other is compressed in the length direction or vise versa. This causes opposite polarization or voltage output $V$ in the two piezoelectric layers. With the electrodes

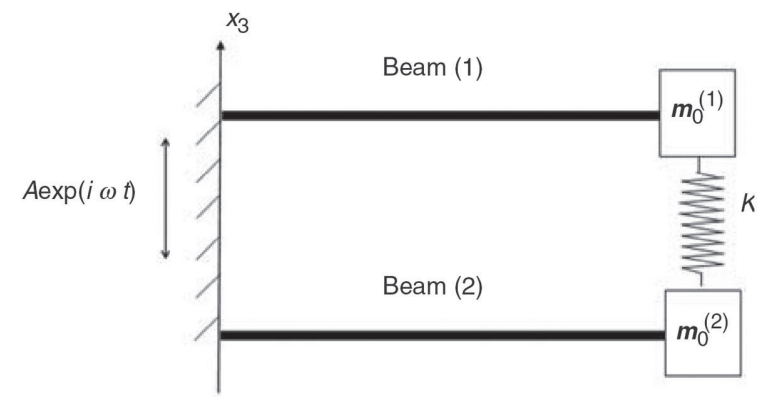

Figure 1. Two beams with end masses elastically connected at their ends.
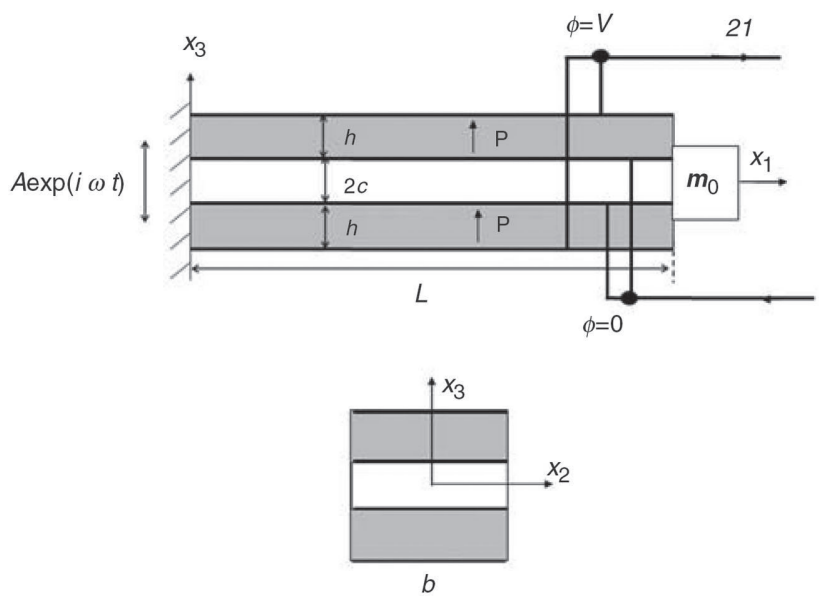

Figure 2. Side view and cross section of one bimorph beam. properly connected, an output current $2 I$ will result. In the following, quantities associated with beams (1) and (2) will carry a superscript 1 or 2 in parenthesis.

\section{Governing Equations}

Under the vertical vibration of the wall, the bimorph beams are essentially in flexural vibrations in the $x_{3}$ direction. For a typical beam the flexural motion $u_{3}$ is governed by (Jiang et al., 2005)

$$
-D u_{3,1111}=m \ddot{u}_{3^{\prime}}
$$

where $D$ is the beam bending stiffness and $m$ is the mass per unit length of the beam. They are given by (Jiang et al., 2005)

$$
\begin{aligned}
& D=\left\{\frac{2}{3} E c^{3}+\frac{2}{3} s_{11}^{-1}\left[(c+h)^{3}-c^{3}\right]\right\} b, \\
& m=\rho 2 c b+2 \rho^{\prime} h b,
\end{aligned}
$$

where $E$ is the Young's modulus of the isotropic elastic layer. $s_{11}$ is the elastic compliance at constant electric field of the piezoelectric layers (Jiang et al., 2005). $\rho$ and $\rho^{\prime}$ are the mass densities of the elastic and piezoelectric layers. The beam bending moment $M$ is given by (Jiang et al., 2005)

$$
M=\int x_{3} T_{1} \mathrm{~d} x_{2} \mathrm{~d} x_{3}=-D u_{3,11}+s_{11}^{-1} d_{31} \frac{V}{h} 2 G
$$

where $V$ is the voltage across each of the two piezoelectric layers. $d_{31}$ is the relevant piezoelectric constant, and

$$
G=\left(c+\frac{h}{2}\right) h b
$$

The transverse shear force in the beam is related to the bending moment and the flexural displacement by (Jiang et al., 2005)

$$
N=\int T_{13} \mathrm{~d} x_{2} \mathrm{~d} x_{3}=M_{, 1}=-D u_{3,111}
$$

We note that $N$ is usually used for the axial force in a beam in extension, and $Q$ is for the shear force in bending. In this article we reserve $Q$ for the material quality factor and, therefore, we are using $N$ for the shear force in bending. This notation is the same as the one used in Jiang et al. (2005). At the cantilevered left end of a beam the boundary conditions are

$$
\begin{aligned}
& u_{3}(0, t)=A \exp (i \omega t) \\
& u_{3,1}(0, t)=0
\end{aligned}
$$


At the right end there is no bending moment:

$$
M(L, t)=0
$$

The electric charge on the top electrode at $x_{3}=c+h$ is given by (Jiang et al., 2005)

$$
\begin{aligned}
Q_{e} & =-b \int_{0}^{L} D_{3}\left(x_{3}=c+h\right) \mathrm{d} x_{1} \\
& =b\left\{s_{11}^{-1} d_{31}(c+h)\left[u_{3,1}(L, t)-u_{3,1}(0, t)\right]+\bar{\varepsilon}_{33} \frac{V}{h} L\right\},
\end{aligned}
$$

where

$$
\bar{\varepsilon}_{33}=\varepsilon_{33}\left(1-k_{31}^{2}\right), \quad k_{31}^{2}=\frac{d_{31}^{2}}{\left(\varepsilon_{33} s_{11}\right)},
$$

and $\varepsilon_{33}$ is the electric permittivity at constant stress (Jiang et al., 2005). The current flowing out of this electrode is

$$
I=-\dot{Q}
$$

The above equations are valid for each of the two bimorph beams. For the two-beam structure as a whole, electrically we consider the case when the two beams are connected in parallel with the same voltage, but the currents should be summed. When the motion is timeharmonic, the output voltage and current are related by

$$
2\left[I^{(1)}+I^{(2)}\right]=\frac{V}{Z_{L}},
$$

where $Z_{L}$ is the complex impedance of the output circuit. At the right ends of the beams the shear force and the spring force together are responsible for the motion of the end masses. From Newton's second law, for the mass at the right end of the upper beam, we have

$$
-N^{(1)}(L, t)-K\left[u_{3}^{(1)}(L, t)-u_{3}^{(2)}(L, t)\right]=m_{0}^{(1)} \ddot{u}_{3}^{(1)}(L, t) .
$$

Similarly, for the mass at the right end of the lower beam,

$$
-N^{(2)}(L, t)+K\left[u_{3}^{(1)}(L, t)-u_{3}^{(2)}(L, t)\right]=m_{0}^{(2)} \ddot{u}_{3}^{(2)}(L, t) .
$$

\section{Solution}

For a typical beam, either the first or the second, for harmonic motions we use the complex notation

$$
\left\{u_{3}(x), V, Q_{e}, I\right\}=\operatorname{Re}\left\{\left\{U(x), \bar{V}, \bar{Q}_{e}, \bar{I}\right\} \exp (i \omega t)\right\} .
$$

Then Equation (1) becomes

$$
-D U_{, 111}=-\omega^{2} m U, \quad 0<x_{1}<L .
$$

The general solution to Equation (16) can be written as (Boyce and DiPrima, 1986)

$$
U=B_{1} \sin \alpha x_{1}+B_{2} \cos \alpha x_{1}+B_{3} \sinh \alpha x_{1}+B_{4} \cosh \alpha x_{1},
$$

where $B_{1}$ through $B_{4}$ are undetermined constants, and

$$
\alpha=\left(\frac{m}{D} \omega^{2}\right)^{1 / 4} .
$$

Substituting of Equation (17) into Equations (9) and (11), we obtain the complex current as

$$
\begin{aligned}
\bar{I}= & -i \omega b\left\{\frac { d _ { 3 1 } } { s _ { 1 1 } } ( c + h ) \left[B_{1} \alpha \cos \alpha L-B_{2} \alpha \sin \alpha L\right.\right. \\
& \left.+B_{3} \alpha \cosh \alpha L+B_{4} \alpha \sinh \alpha L\right] \\
& \left.+\bar{\varepsilon}_{33} \frac{\bar{V}}{h} L\right\} .
\end{aligned}
$$

Equations (17) and (19) are valid for each beam when proper superscripts (1) or (2) are used. The boundary conditions remain to be satisfied are:

$$
\begin{aligned}
U^{(1)}(0) & =A, \\
U_{, 1}^{(1)}(0) & =0, \\
-D^{(1)} U_{, 11}^{(1)}(L)+\frac{d_{31}^{(1)} \frac{\bar{V}}{s_{11}^{(1)}} 2 G^{(1)}}{h^{(1)}} & =0, \\
U^{(2)}(0) & =A, \\
U_{, 1}^{(2)}(0) & =0, \\
-D^{(2)} U_{, 11}^{(2)}(L)+\frac{d_{31}^{(2)}}{s_{11}^{(2)}} \frac{\bar{V}}{h^{(2)}} 2 G^{(2)} & =0,
\end{aligned}
$$

which represents six equations for the undetermined constants. Substitution of Equations (17) and (19) into Equations (20), (13), (14), and the circuit Equation (12) results in

$$
\begin{aligned}
& B_{2}^{(1)}+B_{4}^{(1)}=A \\
& B_{1}^{(1)} \alpha+B_{3}^{(1)} \alpha=0 \\
& -D\left(-B_{1}^{(1)} \alpha^{2} \sin \alpha L-B_{2}^{(1)} \alpha^{2} \cos \alpha L+B_{3}^{(1)} \alpha^{2} \sinh \alpha L\right. \\
& \left.+B_{4}^{(1)} \alpha^{2} \cosh \alpha L\right) s_{11}^{-1} d_{31} \frac{\bar{V}}{h} 2 G=0, \\
& B_{2}^{(2)}+B_{4}^{(2)}=A \\
& B_{1}^{(2)} \alpha+B_{3}^{(2)} \alpha=0
\end{aligned}
$$




$$
\begin{aligned}
& -D\left(-B_{1}^{(2)} \alpha^{2} \sin \alpha L-B_{2}^{(2)} \alpha^{2} \cos \alpha L+B_{3}^{(2)} \alpha^{2} \sinh \alpha L\right. \\
& \left.+B_{4}^{(2)} \alpha^{2} \cosh \alpha L\right) \\
& s_{11}^{-1} d_{31} \frac{\bar{V}}{h} 2 G=0, \\
& D\left(-B_{1}^{(1)} \alpha^{3} \cos \alpha L+B_{2}^{(1)} \alpha^{3} \sin \alpha L+B_{3}^{(1)} \alpha^{3} \cosh \alpha L\right. \\
& \left.+B_{4}^{(1)} \alpha^{3} \sinh \alpha L\right) \\
& +K\left(B_{1}^{(2)} \sin \alpha L+B_{2}^{(2)} \cos \alpha L+B_{3}^{(2)} \sinh \alpha L+B_{4}^{(2)} \cosh \alpha L\right) \\
& +\left(m_{0}^{(1)} \omega^{2}-K\right)\left(B_{1}^{(1)} \sin \alpha L+B_{2}^{(1)} \cos \alpha L+B_{3}^{(1)} \sinh \alpha L\right. \\
& \left.+B_{4}^{(1)} \cosh \alpha L\right)=0, \\
& D\left(-B_{1}^{(2)} \alpha^{3} \cos \alpha L+B_{2}^{(2)} \alpha^{3} \sin \alpha L+B_{3}^{(2)} \alpha^{3} \cosh \alpha L\right. \\
& \left.+B_{4}^{(2)} \alpha^{3} \sinh \alpha L\right) \\
& +K\left(B_{1}^{(1)} \sin \alpha L+B_{2}^{(1)} \cos \alpha L+B_{3}^{(1)} \sinh \alpha L+B_{4}^{(1)} \cosh \alpha L\right) \\
& +\left(m_{0}^{(2)} \omega^{2}-K\right)\left(B_{1}^{(2)} \sin \alpha L+B_{2}^{(2)} \cos \alpha L+B_{3}^{(2)} \sinh \alpha L\right. \\
& \left.+B_{4}^{(2)} \cosh \alpha L\right)=0, \\
& \left.\quad\left[\begin{array}{c}
\left(B_{1}^{(1)}+B_{1}^{(2)}\right) \alpha \cos \alpha L \\
-\left(B_{2}^{(1)}+B_{2}^{(2)}\right) \alpha \sin \alpha L \\
+\left(B_{3}^{(1)}+B_{3}^{(2)}\right) \alpha \cosh \alpha L \\
+\left(B_{4}^{(1)}+B_{4}^{(2)}\right) \alpha \sinh \alpha L
\end{array}\right]+2 \bar{\varepsilon}_{33} \frac{\bar{V}}{h} L\right\} \\
& +2 i \omega b\left\{\frac{d_{31}}{s_{11}}(c+h)\right. \\
& \left.\quad \frac{\bar{V}}{Z_{L}}, \quad \begin{array}{c}
(21) \\
\end{array}\right]
\end{aligned}
$$

which are nine equations for $B_{1}^{(1)}$ through $B_{4}^{(1)}, B_{1}^{(2)}$ through $B_{4}^{(2)}$, and $V$. They are solved over a computer. With the complex notation, the output electrical power is given by

$$
P=\frac{1}{2}\left[\left(\bar{I}^{(1)}+\bar{I}^{(2)}\right) \bar{V}^{*}+\left(\bar{I}^{(1)}+\bar{I}^{(2)}\right)^{*} \bar{V}\right]
$$

where an asterisk represents complex conjugate. For miniaturized power harvesters, the power density, defined as the output power per unit volume, serves as an important measure for device performance:

$$
p=\frac{P}{2 b(2 c+2 h) L} .
$$

\section{Numerical Results}

For a numerical example, we use PZT-5H (Jiang et al., 2005) for the piezoelectric layers which has a mass density $\rho=7500 \mathrm{~kg} / \mathrm{m}^{3}$ and the following elastic, piezoelectric, and dielectric constants (Jiang et al., 2005):

$$
\begin{aligned}
& s_{11}=16.5 \times 10^{-12} \mathrm{~m}^{2} / \mathrm{N}, \\
& d_{31}=-274 \times 10^{-12} \mathrm{C} / \mathrm{N}, \\
& \varepsilon_{11}=3130 \varepsilon_{0}, \quad \varepsilon_{33}=3400 \varepsilon_{0},
\end{aligned}
$$

where $\varepsilon_{0}$ is the electric permittivity of free space.

Damping is included by allowing the elastic constants to assume complex values (Jiang et al., 2005). In our numerical calculations, the real elastic constant $s_{11}$ is replaced by $s_{11}\left(1-i Q^{-1}\right)$, where $Q$ is the quality factor of the material, a large and real number. For ceramics $Q$ is of the order of $10^{2}-10^{3}$. We fix $Q$ to be $10^{2}$ which is chosen to be relatively small and is a representation of the damping of the whole structure. $L=25 \mathrm{~mm}, b=8 \mathrm{~mm}$, $h=2 \mathrm{~mm}, c=2 \mathrm{~mm}$, and the acceleration amplitude $\omega^{2} A$ $=1.0 \mathrm{~m} / \mathrm{s}^{2}$ are fixed in all calculations. The elastic layer is taken to be aluminum alloy with Young's modulus $E$ $=70 \mathrm{GPa}$ and mass density $\rho=2700 \mathrm{~kg} / \mathrm{m}^{3}$. We use the following reference spring stiffness and reference impedance as units:

$$
K_{0}=\frac{3 E I_{0}}{L^{3}}, \quad Z_{0}=\frac{1}{i \omega C_{0}},
$$

where

$$
I_{0}=\frac{b(2 h+2 c)^{3}}{12}, \quad C_{0}=\frac{\bar{\varepsilon}_{33} b L}{h} .
$$

We plot, in Figure 3, the output power density versus the driving frequency for a fixed load impedances $Z_{L}$ / $\left(i Z_{0}\right)=1$ and a fixed spring constant $K=0.05 K_{0}$. Three cases of different end mass ratios are shown. Typically there are two resonant peaks. The distance between the two peaks is very sensitive to the mass ratio. When the

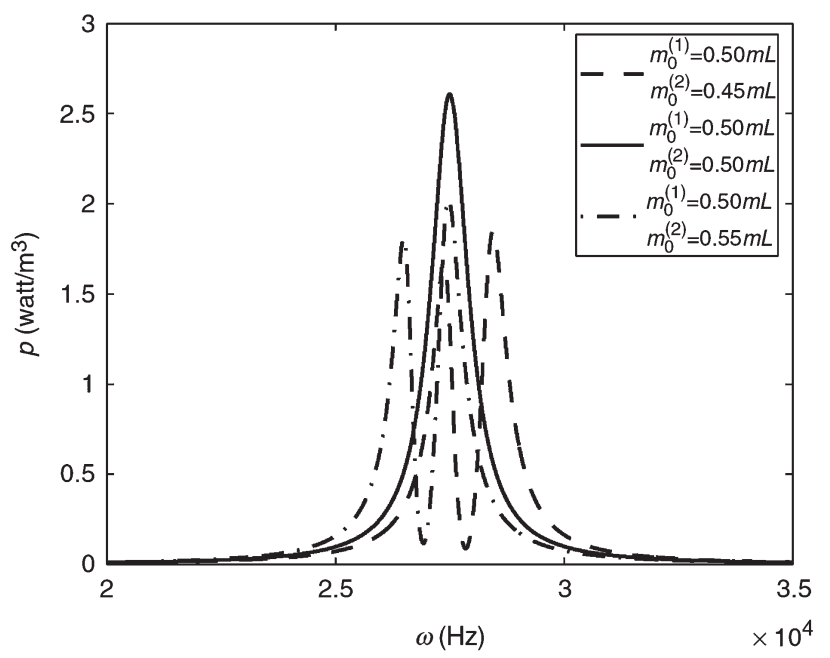

Figure 3. Power density vs frequency for different end masses $(K=$ $0.05 \mathrm{~K} 0)$. 


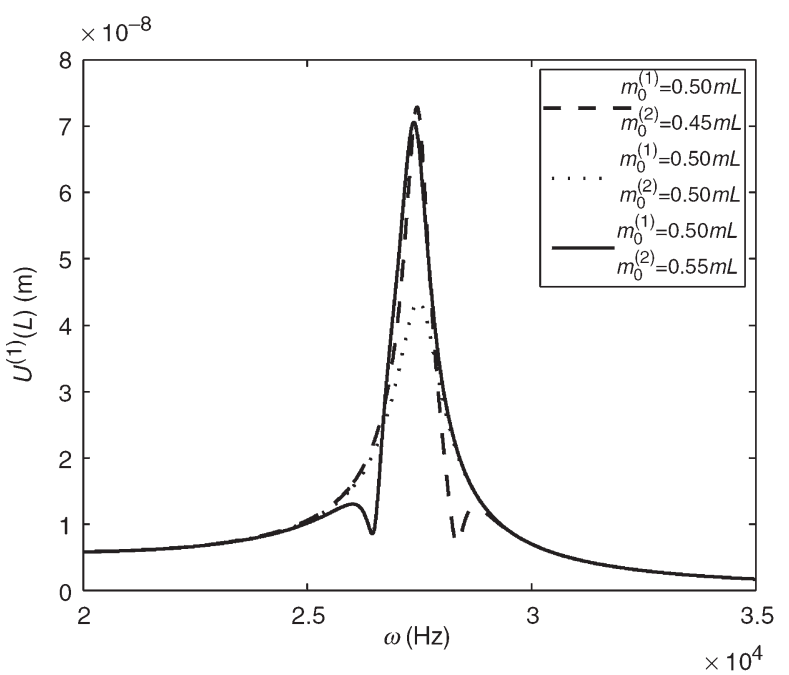

Figure 4. End displacement (absolute value) of Beam (1) vs frequency for different end masses $\left(K=0.05 K_{0}\right)$.

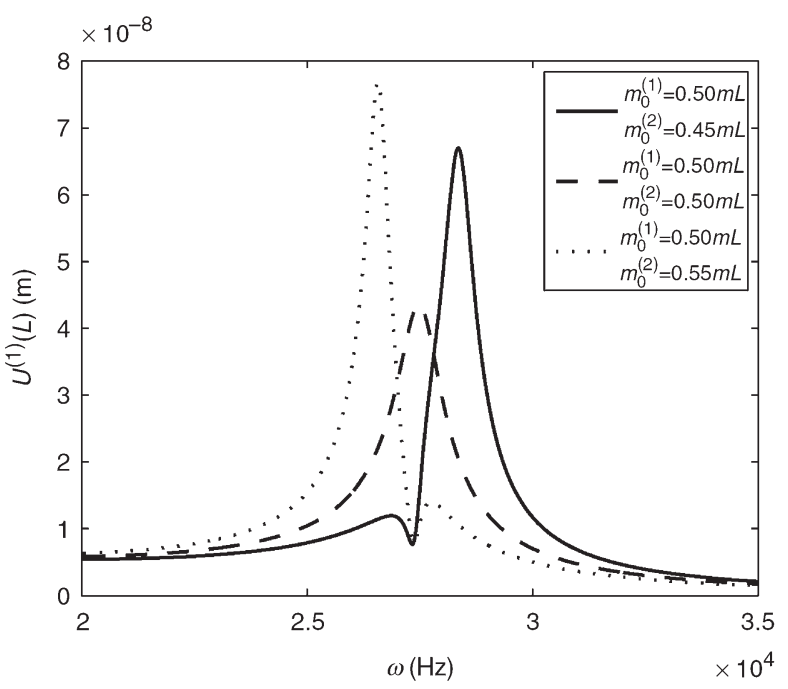

Figure 5. End displacement (absolute value) of Beam (2) vs frequency for different end masses $\left(K=0.05 K_{0}\right)$.

two masses are close, the two peaks are close, forming a wide-band frequency window within which we have significant output. In the special case when the two masses are the same, the two peaks merge into one. For the three cases calculated, the absolute values of the end displacements of the two beams are shown in Figures 4 and 5, respectively, versus the frequency. The amplitude of the end displacement is sensitive to the mass ratio. Since the end mass of Beam (1) is fixed and that of Beam (2) is varied, the resonance varies considerably in Figure 5 but not in Figure 4 .

Figure 6 shows the output power density versus the driving frequency for a fixed load impedances $Z_{L} /\left(i Z_{0}\right)$ $=1$ and fixed mass ratios $m_{0}^{(1)}=0.5 \mathrm{~mL}, m_{0}^{(2)}=0.45 \mathrm{~mL}$, but the spring constant is varied. Three cases of different spring constant are shown. Both the amplitude and the location of the two resonant peaks are sensitive to the spring constant. For the three cases calcu-

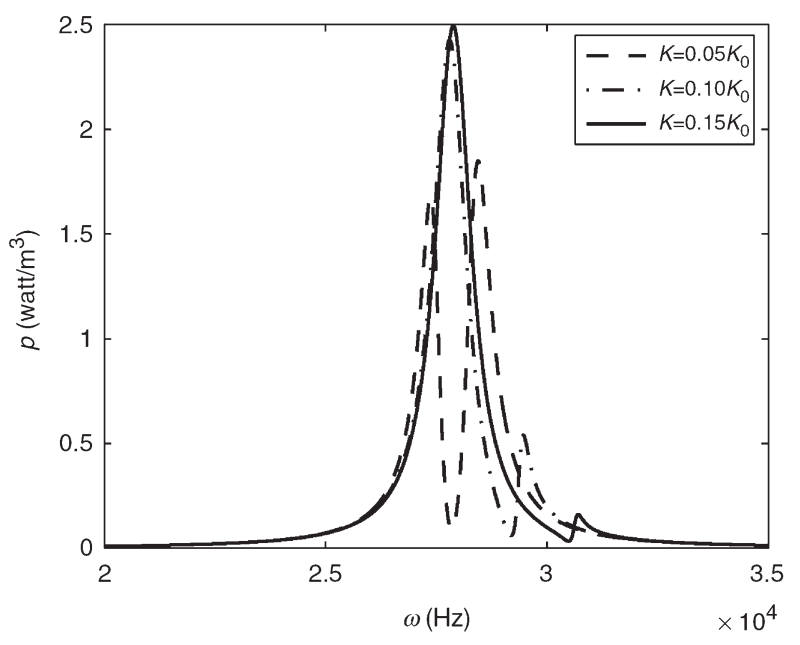

Figure 6. Power density vs frequency for different spring stiffness $\left(m_{0}^{(1)}=0.5 \mathrm{~mL}, m_{0}^{(2)}=0.45 \mathrm{~mL}\right)$.

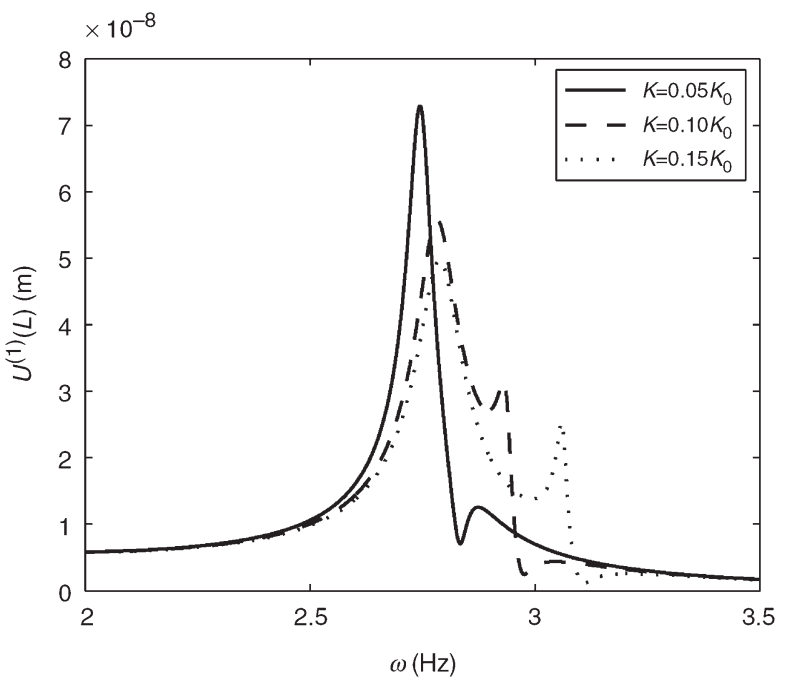

Figure 7. End displacement (absolute value) of Beam (1) vs frequency for different spring stiffness $\left(m_{0}^{(1)}=0.5 \mathrm{~mL}, m_{0}^{(2)}=0.45 \mathrm{~mL}\right)$.

lated, the absolute values of the end displacements of the two beams are shown in Figures 7 and 8, respectively, versus the frequency. Both the amplitude and the location of the resonant peaks are sensitive to the spring constant.

Finally, in Figure 9 we plot the case without a spring between the two beams $(K=0)$ using the same mass ratios as in Figure 3. The solid line in Figure 9 is the same as the solid line in Figure 3 when the two masses are identical because in this case the two beams vibrate in the same way and do not feel the spring. However, the dashed line and the dotted line in Figure 9 are significantly different from the corresponding ones in Figure 3. Basically, the dashed line and the dotted line in Figure 9 represent relatively high and narrow peaks, while the ones in Figure 3 are relatively wide and low peaks. Therefore, the spring makes a significant difference and introduces an important design parameter. 


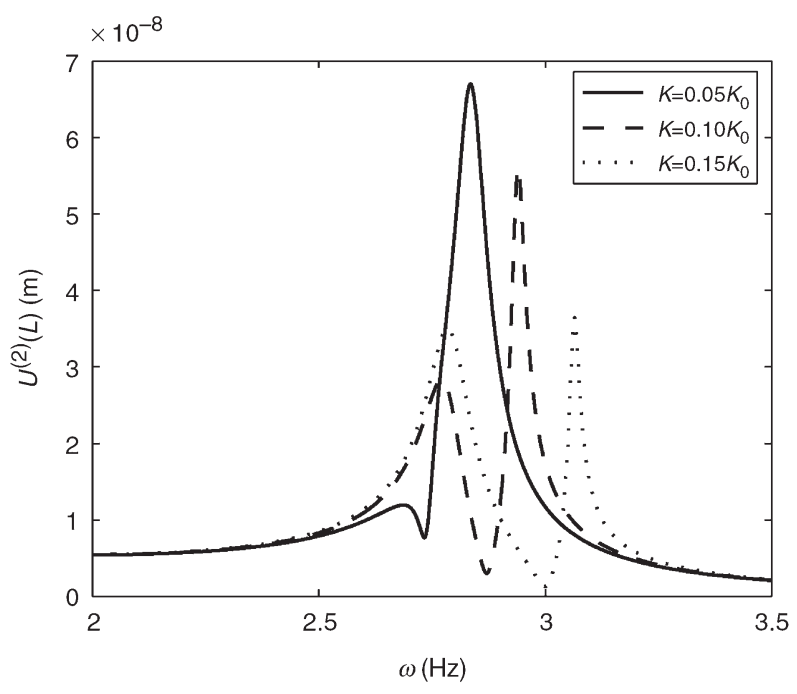

Figure 8. End displacement (absolute value) of Beam (2) vs frequency for different spring stiffness $\left(m_{0}^{(1)}=0.5 \mathrm{~mL}, m_{0}^{(2)}=0.45 \mathrm{~mL}\right)$.

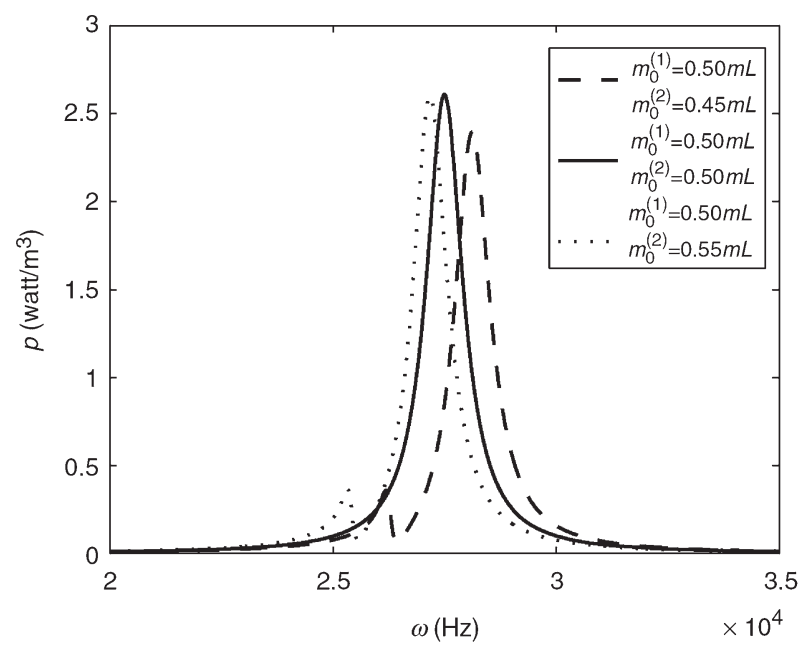

Figure 9. Power density vs frequency for different end masses $(K=0)$.

\section{Conclusion}

When two piezoelectric beam power harvesters with resonant frequencies close to each other are connected electrically by a circuit and elastically by an end spring, wide-band output can be achieved. At the same time the output resonance peaks become lower. The amplitude and location of the resonances are sensitive to the end spring and end masses. Therefore, the performance of the device can be optimized through proper design. This idea can be generalized to connecting more than two harvesters for wider bands.

\section{Acknowledgments}

The first author was supported by a scholarship from the Ministry of Education of China as a visiting student to the University of Nebraska-Lincoln.

\section{References}

Anton, S.R. and Sodano, H.A. 2007. "A Review of Power Harvesting Using Piezoelectric Materials (2003-2006)," Smart Mat. Struct., 16:R1-R21.

Boyce, W.E. and DiPrima, R.C. 1986. Elementary Differential Equations and Boundary Value Problems, 4th edn, John Willey and Sons, New York, p. 263.

Cho, Y.S., Pak, Y.E., Han, C.S. and Ha, S.K. 2000. "Fiver-port Equivalent Electric Circuit of Piezoelectric Bimorph Beam," Sen. Actuat., 84:140-148.

Engel, T.G., Keawboonchuay, C. and Nunnally, W.C. 2000. “Energy Conversion and High Power Pulse Production Using Miniature Piezoelectric Compressors," IEEE Trans. Plas. Sci., 28: 1338-1341.

Ha, S.K. 2001. "Analysis of the Asymmetric Triple-Layered Piezoelectric Bimorph Using Equivalent Circuit Models," J. Acoust. Am., 110:856-864.

Hu, Y.T., Hu, H.P. and Yang, J.S. 2006. "A Low-frequency Piezoelectric Power Harvester Using a Spiral-shaped Bimorph," Sci. China G, 49:649-659.

Hu, H.P., Xue, H. and Hu, Y.T. 2007a. "A Spiral-shaped Harvester with an Improved Harvesting Element and an Adaptive Storage Circuit," IEEE Trans. Ultrason. Ferroelect. Freq. Contr., 54: 1177-1187.

Hu, H.P., Cui, Z.J. and Cao, J.G. 2007b. "Performance of a Piezoelectric Bimorph Harvester with Variable Width," J. Mech., 23(3):197-202.

Hu, Y.T., Xue, H. and Hu, H.P. 2007c. "A Piezoelectric Power Harvester with Adjustable Frequency through Axial Preloads," Smart Mater. Struct., 16:1961-1966.

Hu, H.P., Zhao, C., Feng, S.Y. and Hu, Y.T. 2008. "Adjusting the Resonant Frequency of a PVDF Bimorph Power Harvester Through a Corrugation-shaped Harvesting Structure," IEEE Trans. Ultrason. Ferroelect. Freq. Contr., 55:668-675

Jiang, S.N., Li, X.F., Guo, S.H., Hu, Y.T., Yang, J.S. and Jiang, Q. 2005. "Performance of a Piezoelectric Bimorph for Scavenging Vibration Energy," Smart Mat. Struct., 14:769-714.

Jiang, S.N., Jiang. Q., Hu, Y.T., Li, X.F., Guo, S.H. and Yang, J.S. 2006. "Analysis of a Piezoelectric Ceramic Shell in Thicknessshear Vibration as a Power Harvester," Int. J. Appli. Electromag. Mech., 24:25-31.

Jiang, S.N. and Hu, Y.T. 2007. “Analysis of a Piezoelectric Bimorph Plate with a Central-attached Mass as an Energy Harvester," IEEE Trans. Ultrason. Ferroelect. Freq. Contr., 54:1463-1469.

Roundy, S., Write, P.K. and Rabaey, J. 2003. "A Study of Low Level Vibrations as a Power Source for Wireless Sensor Nodes," Comp. Commun., 26:1131-1144.

Taylor, G.W., Burns, J.R., Kammann, S.A., Powers, W.B. and Welsh, T.R. 2001. "The Energy Harvesting Eel: A Small Subsurface Ocean/River Power Generator," IEEE J. Ocean. Eng., 26:539-547.

Yang, J.S., Zhou, H.G., Hu, Y.T. and Jiang, Q. 2005. "Performance of a Piezoelectric Harvester in Thickness-stretch Mode of a Plate," IEEE Trans. Ultrason. Ferroelect. Freq. Contr., 52: 1872-1876.

Yang, J.S., Chen, Z.G. and Hu, Y.T. 2007. “An Exact Analysis of a Rectangular Plate Piezoelectric Generator," IEEE Trans. Ultrason. Ferroelect. Freq. Contr., 54:190-195. 